\title{
Fuzzy Optimization Model for post harvest selection process of Pecan (Caryaillinoinensis)
}

\author{
Consuelo Espino, Ciro Rodríguez, Doris Esenarro
}

\begin{abstract}
The quality of food is associated with a set of properties and characteristics that can be considered from its chemical and nutritional physical composition that have the ability to meet the needs of the consumer. The paper aims to evaluate the application of fuzzy logic in the evaluation and classification of the selection of pecans in the post harvest process using tests and instruments that determine their best quality. Fuzzy logic has proven to be very effective with matlab/simulink to develop and simulate the entire system, through an appropriate choice of rules and membership functions and applying the Mamdani method to defuzzification the results considering they are positive.
\end{abstract}

Keywords: Fuzzy logic, matlab/simulink, post-harvest, selection process, sensory acceptability, caryaillinoinensis.

\section{INTRODUCTION}

Pecan producers are aware that guaranteeing quality products and in accordance with customer requirements is not only possible with the fuzzy logic model in the system of their internal processes. Computer modeling and simulation allows to support and facilitate the evaluation of a set of parameters for the study and analysis of the selection of pecans.

Zadeh [1] was the first to disseminate the novel way of characterizing non-probabilistic uncertainties, which he called fuzzy logic (FL), which embodies the nature of the human being's mind and which contrasts with the traditional Boolean logic. A diffuse system is based on a diffuse set, membership or diffuse membership and diffuse variables. The system consists of a fuzifier, a knowledge base (base rules), an interference engine and a defuzifier. The knowledge base is a collection of fuzzy "if-else" rules. The term FL denotes a modeling approach, where the functional dependencies between the input and output variables are described, through the set of rules and following the reasoning with the intersection (AND), union (OR) and negation (NOT) operators.

In [2], since the 1990s, the Fuzzy Logic has had applications in decision theory, control systems and the delimitation of operating system behavior profiles; especially in the areas of electrical engineering, artificial intelligence, strategic planning and; more recently, in information sciences. The Fuzzy Logic methodology allows ambiguity to work, opening a perspective of a quantitative alternative structure, which replaces the Aristotelian logic (is or is not) with Bertrand Russell's logic; in which uncertain statements may have relevant values between the values of zero and 1, where in this paradigm, the extremes represent the absence and fullness of relevance, respectively.

In the sensory evaluation, the food industry has a tool that allows it to assess the perception - by the consumer of a product as a whole, or of a specific aspect of it. In this type of tests, the information provided by a panel is perceived by the sensory organs from sight, smell, hearing, taste and touch and the results allow to determine how the processing and formulation of a product affects the acceptability of a food. Sensory evaluations require thorough organization. It begins with the selection of the attributes to be categorized in the sample, the design of the instruments for the collection of information, the type of test to be performed and the determination of the type and number of panelists that will participate in the evaluation The selection of attributes is due to the nature of the food and the recipient of the product. On the other hand, the design of the instrument will be in correspondence with the type of test to be carried out, and finally, the number of panelists necessary for a sensory test to be valid will depend on the type of judge that will be employed.

They also maintain that the diffuse implementation in food quality control for the food industry has been the focus of different researchers who have designed specific applications for this task, where the reasoning process is expressed in linguistic terms of operators and experts. They further mention that the application of linguistic decision analysis for sensory evaluation can be used to systematically model and manage uncertainty and inaccuracy of information in this type of problem. They consider the FL as an important decision making tool, to compare a product developed with similar products available in the market, which can be used to find out the reasons and high ranking of products evaluated by the judges and to determine the importance of individual factors in the overall quality of a product, according [3] the most important factors for a particular market can be identified and improved, which contributes to the development and improvement of products.

The use of fuzzy logic in the pecan selection process is of particular importance since it is possible to minimize the effect of any characteristic when it reaches a value immediately outside its established range. 


\section{ILLINOINENSIS)}

\section{MATERIALS AND METHODS}

On the materials and method, the following were taken into account:

Data origin: Classification form of the pecan "with shell" by its size or caliber as shown in Table I.

Table 1. Classification for size

\begin{tabular}{|l|r|r|}
\hline Size or gauge & $\begin{array}{c}\text { Number of pecans } \\
\text { per kilogram }\end{array}$ & $\begin{array}{c}\text { Number of pecans } \\
\text { per pound * }\end{array}$ \\
\hline Giant & 121 or less & 55 or less \\
\hline Extra big & $123-139$ & $56-63$ \\
\hline Big & $140-170$ & $64-77$ \\
\hline $\begin{array}{l}\text { Means, } \\
\text { medium }\end{array}$ & $171-210$ & $78-95$ \\
\hline Small & $211-264$ & $96-120$ \\
\hline
\end{tabular}

Classification form by edible content of the pecan "with shell"

According the percentages of edible content of the pecan "with shell" are established in the following Table II, by Category and Type:

Table 2. Classification for category

\begin{tabular}{|l|l|l|}
\hline Category & \multicolumn{1}{|c|}{$\begin{array}{c}\text { Type I: Creole } \\
\text { Pecan }\end{array}$} & \multicolumn{1}{c|}{$\begin{array}{c}\text { Type II: Variety } \\
\text { pecan }\end{array}$} \\
\hline I & - & $>54$ \\
\hline II & $>40$ & $<54->50$ \\
\hline III & $<40->30$ & $<50->45$ \\
\hline
\end{tabular}

Defects tolerance form of the "pecan with shell" according to quality categories.

Table 3. Classification for defects

\begin{tabular}{|l|l|l|l|}
\hline \multicolumn{1}{|c|}{ Defects } & Cat. I & Cat. Ii & \multicolumn{1}{c|}{$\begin{array}{c}\text { Cat. } \\
\text { Iii }\end{array}$} \\
\hline External Defects & 6 & 12 & 25 \\
\hline Shape & 5 & 10 & 15 \\
\hline Broken & 4 & 7 & 10 \\
\hline Cracked Or Cracked Shell & 2 & 5 & 8 \\
\hline Stains And Color Defects & 5 & 8 & 10 \\
\hline Pecan Fragments & 3 & 5 & 10 \\
\hline Empty Units & 0,5 & 1 & 1,5 \\
\hline Foreign Matters & 1 & 5 & 10 \\
\hline $\begin{array}{l}\text { Total Tolerance Of External } \\
\text { Defects }\end{array}$ & 6 & 12 & 25 \\
\hline \multicolumn{1}{|c|}{ Other Defects } & Cat. I & Cat. Ii & Cat. Iii \\
\hline $\begin{array}{l}\text { Rancidity (Peroxide Index In } \\
\text { Meq / Kg) }\end{array}$ & 0 & $>0-<5$ & \multicolumn{2}{c|}{$<10$} \\
\hline
\end{tabular}

Boards obtained by Agrifood-Quality and Health Service (Senasa).

\section{Diffuse Logic:}

Basically, Diffuse Logic is a multivariate logic that allows to represent mathematically uncertainty and vagueness, providing formal tools for its treatment. As Zadeh indicates, "When complexity increases, precise statements lose their meaning and useful sentences lose precision.", Which can be summed up as "trees don't let you see the forest." Basically, any problem in the world can be solved as given a set of input variables (input space), obtaining an adequate value of output variables (output space). La lógicadifusapermiteestablecerestemapeo de una forma adecuada, atendiendo a criterios de significado (y no de precisión). The term Fuzzy Logic was first used in 1974. It is currently used in a broad sense, grouping fuzzy set theory, if-then rules, fuzzy arithmetic, quantifiers, etc.

Diffuse Controller:

Fuzzy logic is mainly applied in fuzzy control systems that use ambiguous expressions to formulate rules that control the system. A diffuse control system works very differently than conventional control systems.

They use the knowledge of an expert to generate a knowledge base that will give the system the ability to make decisions about certain actions that arise in its operation.

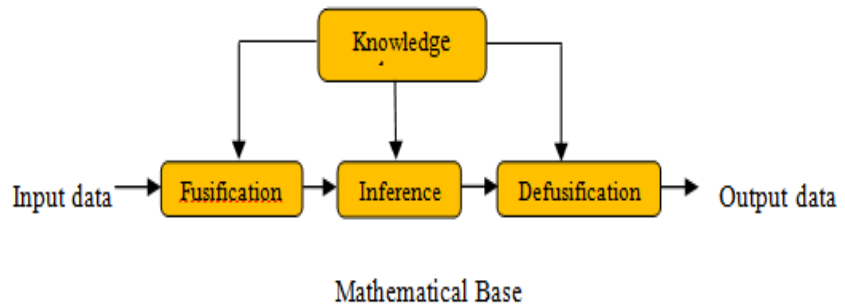

Figure 1. Fuzzy controller has a defined structure.

Fuzification:

The merger aims to convert real values into fuzzy values. In the merger, degrees of belonging are assigned to each of the input variables in relation to the fuzzy sets previously defined using the membership functions associated with fuzzy sets.

\section{Knowledge Bases:}

The knowledge base contains the knowledge associated with the application domain and control objectives. At this stage, the linguistic control rules that will make the decisions that will decide how the system should act should be defined.

\section{Inference:}

The inference relates the fuzzy sets of input and output to represent the rules that will define the system. Inference, knowledge base information is used to generate rules through the use of conditions.

\section{Defusification:}

Defusification performs the process of adapting the fuzzy values generated in the inference in values that will later be used in the control process.

\section{Developing:}

For the development of this project we first define our linguistic variables as shown in Figure 2., which in this case are:

- Size

- Weight

- Whole

- Dry

- Healthy

- Clean

- Free of cracks 


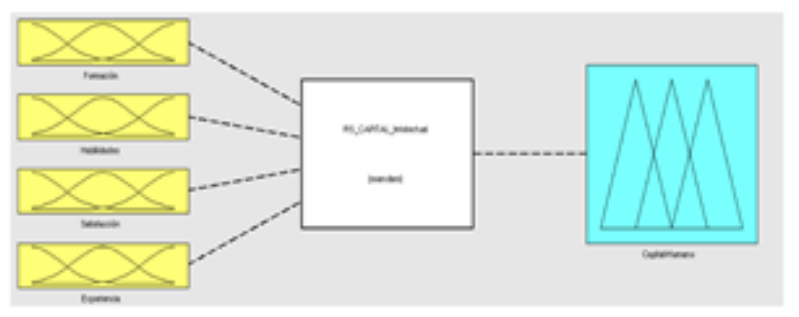

Figure 2. The linguistic variables mentioned above are shown

Once our linguistic variables are defined, Fig. 3, we define the linguistic values:

$\begin{array}{ll}\text { - } & \text { Small } \\ \text { - } & \text { Medium } \\ \text { - } & \text { Bery cold } \\ \text { - } & \text { Cold } \\ \text { - } & \text { High } \\ \text { - } & \text { Very high }\end{array}$

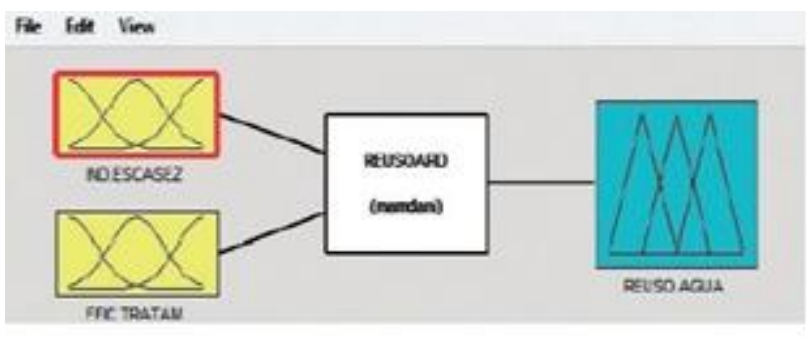

Figure 3. Linguistic values in the model

With the help of a code in Matlab we will generate the working ranges of our system for each of the linguistic values that we introduced above.

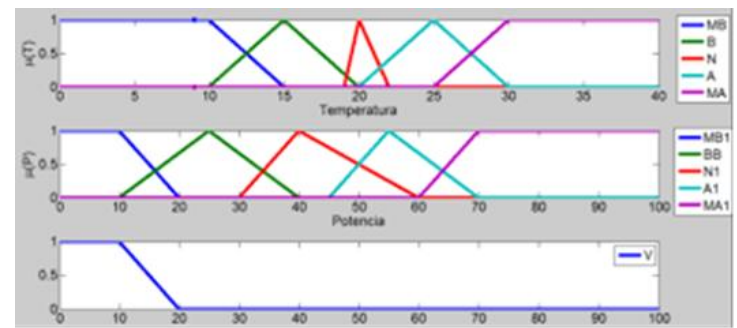

Figure 4. Ranges of linguistic values

Once all these parameters have been defined, we will make our fuzzy rules, as in Table IV, in the form if A and / or B else C. shows the fuzzy rules based on the experience of an expert.

Table 4. Diffuse inference rules

\begin{tabular}{|c|c|c|c|c|}
\hline & Dry & Verycold & Cold & High \\
\hline \multirow{3}{*}{ Size } & small & medium & small & medium \\
\cline { 2 - 5 } & medium & tall & medium & tall \\
\cline { 2 - 5 } & big & tall & tall & tall \\
\hline
\end{tabular}

IDENT toolbox is used to obtain the transfer function of our system and see its behavior with the different fuzzy rules we created earlier, the toolbox environment is shown. For this we use the following code:

$>\mathrm{w}=[]$

$>$ clear all

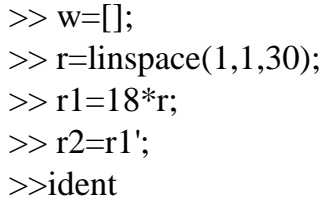

Opening System Identification Tool shows as Figure 5 the estimation data set done.

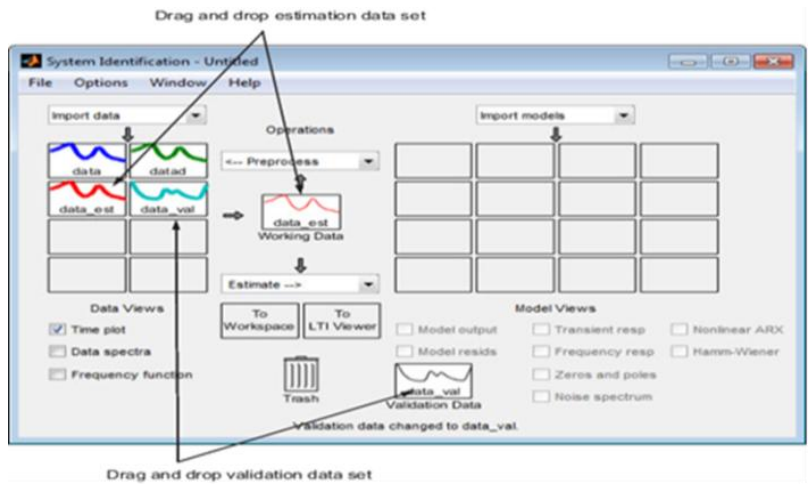

Figure 5. Estimation data set

In this [4] argue that products that are destined for food must meet microbiological, physical and nutritional quality parameters. However, compliance with these aspects is not enough. Its quality will not be fully defined if those characteristics are not added to the organoleptic, and that is where the acceptance of a product is a very important decision factor.

\section{RESULTS}

Once the steps have been completed, we will obtain a graph which shows us the three-dimensional response, as shown in Fig. 6., of our proposed fuzzy model.

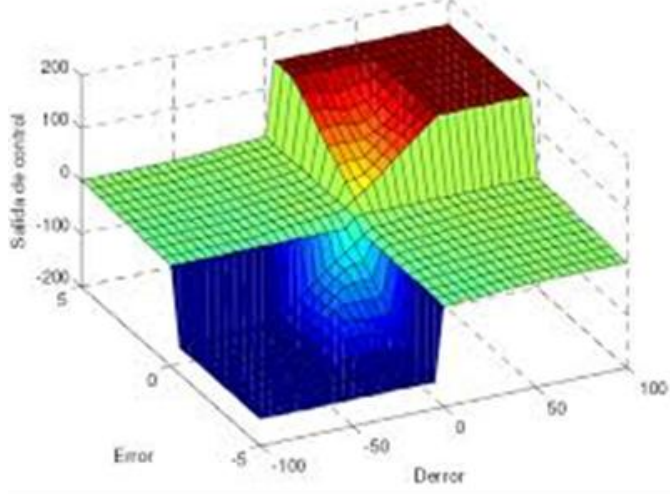

Figure 6. Three-dimensional response

Likewise, in the toolbox we can observe in the simulation, the behavior or variation in time of our fuzzy rules, as shown in Figure 7, with their respective outputs. 


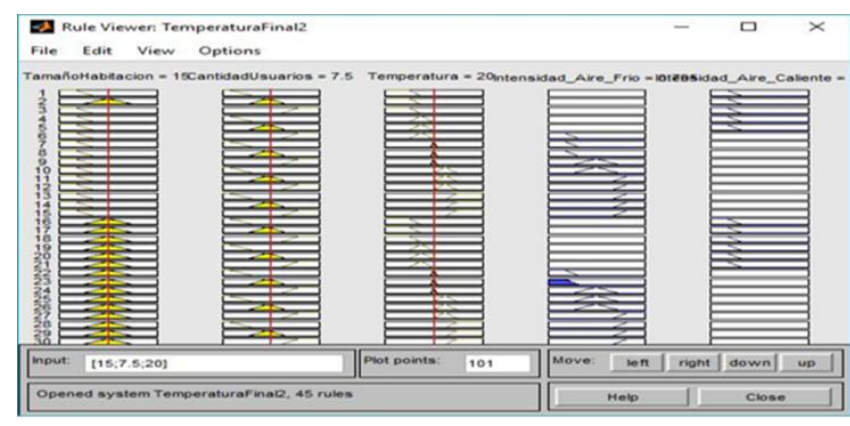

Figure 7. Rules with their respective outputs

With the toolbox Ident we can generate our transfer function by choosing the zeros and poles that our system has, in this way we can check if our system is stable or not stable and likewise, we can observe the behavior or stabilization of our diffuse system.

Finally, we use Mat lab Simulink to represent or simulate our diffuse system: input, controller or plant, output,

This simulation we can graphically observe the response of our system and check if all previously established fuzzy rules are met, the above is shown in Figure 8.

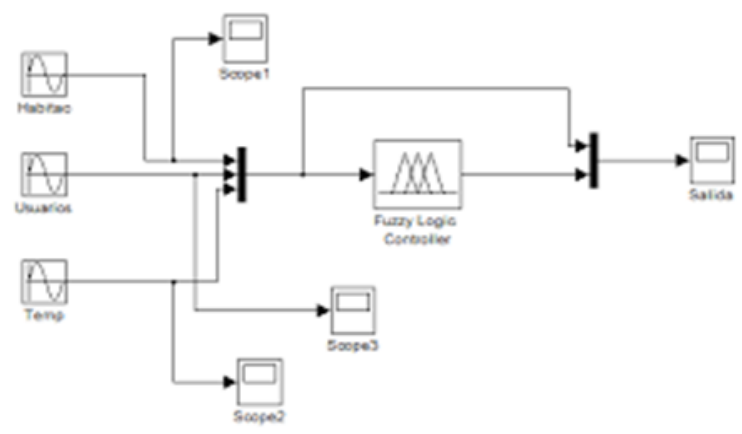

Figure 8. Graphically response of simulation

Sensory analysis is an interdisciplinary science through which panelists using complex interactions of the senses can determine the preference or acceptability of products. Its evaluation is based on response to stimuli whose sensations are: intensity, magnitude, duration, quality, pleasure or discontent. Stimuli can be quantified by physical-chemical methods and sensations by psychological processes [5].

\section{DISCUSSION}

When the problems are posed in a natural language [3], although it could be considered that they are not expressed in a technical way, they contain information that is very rich and that reflects the experience of the data collector [4]. When this empiricism is taken into account, the results obtained are much closer to what happens in reality.

Automatic detection is possible by applying mathematical methods and models, as mentioned in [6, 7,8,9,10,11] the important thing is to capture the situations presented in the real world as also shown by fuzzy logic.

The use of fuzzy systems offers innumerable applications for operations in which sensory criteria are required; one of those applications has been demonstrated in the system proposed in this research.

In order for the diffuse system to operate efficiently, it is of the utmost importance to select an appropriate selection panel [4], through which the quality standards of the different attributes in a given product will be obtained, thus representing the requirements of the consumer.

\section{ACKNOWLEDGEMENTS}

A special acknowledgment to professorCiro Rodriguez involved in the Artificial Intelligence field for his unconditional support to realize this paper.

\section{REFERENCES}

1. Zadeh, L.A. Fuzzy Sets. Information and Control 8: 338-353). (1965)

2. Lanzillotti, R.S.; Lanzillotti, H.S. Sensory analysis on the decision of fuzzy decision. Rev. Nutr., Campinas, 122: 145-157. (1999)

3. Mukhopadhyay, S.; Majumdar, G.C.; Goswami, T.K.; Mishra, H.N. Fuzzy logic (similarity analysis) approach for sensory evaluation of chhanapodo. LWT - Food Science and Technology 53: 204-210. (2013)

4. Ávila-de Hernández, R.; González-Torrivilla, C. The sensory evaluation of fruit-based drinks: a diffuse approach. University, Science and Technology 15: 171-182. (2011)

5. Cavalcanti, M .; da Silva, F .; Cavalcanti, J .; Florentino, E .; Florêncio, I .; Moreira, R. Applicação da logic fuzzy na sensory analysis of pão enriched. Brazilian Journal of Agricultural and Environmental Engenharia 17: 208-215., (2013)

6. Lenin Vera-Montenegro, AmparoBaviera-Puig, Jose-Maria Garcia-Alvarez-Coque, Selection of cocoa post-harvest technology using fuzzy logic, fuzzy economic review vol. xix, no. 2, nov. 2014, p. 3-19

7. Mirza Abdul WarisBegh, Zhenbin Zhang, SiddharthAgarwal (2017), Predictive Current Control of Modular Multilevel Converter for HVDC system with Online weighting factor tuning using Fuzzy Logic.

8. HawafAbdAlhakim, O.E. Emam, AmrAtifAbdEI-Mageed, A Computational Model for Multi-level Quadratically Constrained Quadratic Optimization under Fully Fuzzy Environment, Journal of Advances in Mathematics and Computer Science Art. 42263, 27(5) : 1- 21, 2018

9. Marcel Ioan Bolos, Ioana Alexandra Bradea and CameliaDelcea, A fuzzi logic algorithm for optimizing the investment decisions within companies, Symmetry 2019,vol. 11,186

10. P. ArunaKumari, I. SantiPrabha Network Selection in Heterogeneous Wireless Environment using Computationally Reduced Fuzzy Rule Base System ,International Journal of Recent Technology and Engineering (IJRTE) ISSN: 2277-3878, Volume-7, Issue-5S4, February 2019

11. José B., Ciro R., Doris E. "Real Time Facial Expression Recognition System Based on Deep Learning". International Journal of Recent Technology and Engineering (IJRTE) ISSN: 2277-3878, Volume-8, Issue-2S11, September 2019

\section{AUTHORS PROFILE}

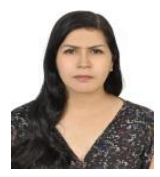

\section{Consuelo Elsa, EspinoVillar}

Doctorate candidate of Systems Engineering of the National University Federico Villarreal, with experience in Software, Systems Analyst.

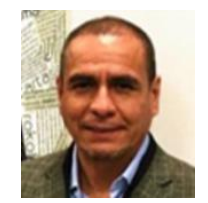

\section{Ciro Rodriguez Rodriguez}

Professor at the School of Software Engineering at the National University Mayor de San Marcos and also at the Computer Science School and Graduate School of the National University Federico Villarreal, with science studies at the Abdus Salam International Center for Theoretical Physics (ICTP) and the United States Particle Accelerator School (USPAS). 
Professor at the Faculty of Environmental Engineering and Graduate School of the National University Federico Villarreal, with studies in System Engineering, Architecture and Environmental Engineering. 